\title{
Prevalence, awareness, and associated risk factors of hypertension in older adults in Africa: a systematic review and meta- analysis protocol
}

\author{
William K. Bosu ${ }^{1,3^{*}}$, Justice M. K. Aheto ${ }^{2}$, Eugenio Zucchelli $i^{3}$ and Siobhan Reilly ${ }^{3}$
}

\begin{abstract}
Background: The health of older persons has not been a major priority in many African countries. Hypertension is one of the common health problems of older persons. However, there is little information on the prevalence of hypertension in older adults in Africa. This is in spite of the fact that Africa has the highest age-standardized prevalence of hypertension in the world. We therefore present this protocol to conduct a systematic review and meta-analysis on the prevalence of hypertension and the level of its awareness among older persons living in Africa.

Methods: Major databases (EMBASE, MEDLINE, Academic Search Complete, CINAHL, PsycINFO) and unpublished literature will be searched to identify population-based studies on hypertension in adults aged 50 years and older living in Africa. Eligible articles are those which use the 140/90-mmHg cutoff to diagnose hypertension and were published from 1980 to present. We will exclude subjects in restricted environments such as patients and refugees. Articles will be independently evaluated by two reviewers to determine if they meet the inclusion criteria. They will also evaluate the quality of included studies using a validated tool by Hoy and colleagues for prevalence studies. The main outcome is the prevalence of hypertension while the explanatory variables include demographic, socio-economic, dietary, lifestyle and behavioural factors. Effect sizes in bivariate and multivariate analyses will be presented as odds or prevalence ratios. We will explore for heterogeneity of the standard errors across the studies, and if appropriate, we will perform a meta-analysis using a random-effects model to present a summary estimate of the prevalence of hypertension in this population.
\end{abstract}

Discussion: The estimates of the prevalence, the risk factors and the level of awareness of hypertension could help in galvanizing efforts at prioritizing the cardiovascular health of older persons in Africa.

Systematic review registration: PROSPERO CRD42017056474

Keywords: Older adults, Hypertension, Risk factors, Determinants, Awareness, Africa

\section{Background}

Hypertension is a major risk factor for cardiovascular diseases, accounting for $52.5 \%$ of all strokes in Africa compared with $38.8 \%$ in the industrialized region [1]. The World Health Organization (WHO) estimates that its African Region had the highest age-standardized prevalence of adult hypertension in the world in 2008

\footnotetext{
* Correspondence: wbosu@wahooas.org

${ }^{1}$ West African Health Organisation (WAHO), 175 Avenue Ouezzin Coulibaly,

01 BP 153, Bobo-Dioulasso, Burkina Faso

${ }^{3}$ Division of Health Research, Faculty of Health \& Medicine, Lancaster

University, Furness Building, Lancaster LA1 4YG, UK

Full list of author information is available at the end of the article
}

(38.1\% in men and $35.5 \%$ in women) [2]. The mean blood pressure levels in sub-Saharan Africa (SSA) increased significantly between 1975 and 2015 to levels that are among the highest in the world [3]. Before-andafter studies as well as estimates from systematic reviews also show significant increases in the prevalence of hypertension in the same rural or urban populations over time in Africa [4-6]. Important features of hypertension in Africa include young age of onset of complications [7], high prevalence of target organ damage [8-11], poor knowledge about the disease in the general population and among hypertensive persons [12, 13], use of 
alternative therapy $[14,15]$, low levels of detection and control [16-18] and clustering of risk factors [19] and co-morbidities [18].

Older persons have higher levels of mean blood pressure and hypertension [20]. For example, a recent systematic review estimated that the pooled prevalence of hypertension in adults aged $65+$ years $(61.0 \%)$ in SSA was 2.5 times that among persons aged 18-64 years (24.4\%) [20]. An epidemiological model showed that the pooled prevalence of hypertension in Africa in the elderly ranges from two to four times than that in 40 years or younger [21]. Older persons aged $50+$ years are more likely than younger adults in urban and rural Africa to have hypertension or to report risk factors for chronic non-communicable diseases (NCDs) [22, 23].

Older persons in Africa suffer multi-morbidity, with a majority having hypertension in addition to other health problems such as angina, arthritis, asthma, cataract, chronic obstructive pulmonary disease, depression, diabetes, edentulism, cognitive impairment, obesity and stroke [24-27]. NCDs, particularly cardiovascular diseases, are the commonest cause of medical admissions among the elderly in Africa [28]. The treatment and control rates of hypertension in older adults in Africa are generally lower than those in high-income countries $[18,29-31]$.

The population of Africa is ageing with the proportion aged 60 years and above $(60+)$ projected to increase from $5.2 \%$ in 2000 to $8.9 \%$ in 2050 and $19.6 \%$ in 2100 [32]. SSA will experience the fastest growth rate from the 1950s to the 2040s in the world [33]. The changing demographics, accompanied by an epidemiologic transition favour an increase in chronic NCDs. The Institute of Health Metrics and Evaluation estimates that the proportion of total deaths due to NCDs in Africa that occurred among persons aged $50+$ years increased from $67.3 \%$ in 1990 to $71.1 \%$ in 2015 [34]. The share of all NCD deaths among those aged $50+$ years also increased from 66.5 to $71.6 \%$ over the same period.

Among persons aged 50-69 years in Africa, the leading risk factors for disease burden, measured in disabilityadjusted life years (DALYs) lost, are high systolic blood pressure (SBP), high body mass index and high fasting plasma glucose. The proportion of deaths attributable to cardiovascular diseases in Africa remained stable at 29.1\% in 1990 and $28.9 \%$ in 2015. The proportion of DALYs attributable to cardiovascular diseases decreased slightly over the same period was from 23.1 to $22.1 \%$. These statistics vary between countries. In Ghana, for example, cardiovascular diseases were the leading health problems in persons aged 50-69 years in 2015 accounting for 30.3 and $24.5 \%$ of total deaths and DALYs lost respectively.

It is not often appreciated that hypertension is preventable even in old age through lifestyle changes [35].
For example, promoting light or moderate physical activity has been shown to reduce mortality and heart attacks in older men [36]. Treatment of hypertension in older adults prevents cardiovascular complications and improves their quality of life [37]. The beneficial impact of lowering blood pressure on cardiovascular disease and mortality is not limited to younger or middle-aged adults [38].

There are several reasons why a systematic review on the prevalence of hypertension in older adults in Africa is needed. While a number of systematic reviews of hypertension have been undertaken at country level [39-42], subregional [19] and continental levels [17, 43, 44] and recently among children and adolescents [45], there is little information among the older adult population in Africa. To date, only one systematic review with meta-analysis of the prevalence of hypertension in older adults in Africa has been published [46]. Unlike our proposed review which defines older age as 50 years and older in line with other studies $[23,31,47]$, the Kaze et al. study used a threshold of 55 years [46] and reported a pooled prevalence of hypertension of $55.2 \%$ among persons aged 55 years and older. There was substantial heterogeneity $\left(I^{2}=95.5 \%\right)$ across the studies analysed, due probably in part to their method of study selection. Our review will analyse studies primarily targeting older persons, whereas their review extracted data pertaining to older persons as a subset from any study of the general population. These differences in study selection could lead to substantial differences in the number and sample sizes in the final set of studies that are analysed. For example, two papers by Addo et al. [48, 49] conducted respectively in rural subjects aged 18-99 years and urban civil servants aged $25-68$ years would not be eligible in our study whereas they contributed three data points from the age groups 55-64 years, $\geq 55$ years and $\geq 65$ years in the Kaze et al. [46] study.

Besides the differences in the set of studies to be analysed, we expect the different study selection methods to yield different study sample sizes, both of which affect heterogeneity across studies. In household surveys in Africa, the older age groups tend to be under-represented. Consistent with this observation, the $55+$ years age group constituted only about $21 \%$ of the total sample size in the Kaze et al. [46] review. As an example of the differences in the sample size to be evaluated, whereas our review on the prevalence of hypertension will include all (100\%) the 3840 South Africans aged 50 years and older in an eligible study [50], the Kaze et al. [46] review included only the age group 60 years and older which represented $55.9 \%$ of the total sample. Further, we expect that the inclusion of studies published prior to the year 2000 will yield additional relevant studies.

Restricting our studies to those that were conducted specifically in older persons will provide a better basis to make policy proposals relating to this age group than 
the Kaze et al. review in which the included studies covered a mix of age groups. A study conducted with the aim to assess the difference in the prevalence of hypertension between formal and informal urban areas in an African city [51] or one that aimed to estimate the prevalence of hypertension and associated risk factors in civil servants [49] would hardly be expected to yield findings that are directly relevant to older persons. Thus, we expect the profile of subjects in the included studies between our review and the published review [46] to be significantly different owing to the differences in the age groups, time period and inclusion criteria.

\section{Review question}

The review questions are as follows:

1. What is the prevalence and trends in hypertension in adults aged 50 years and older living in Africa?

2. What are the factors associated with hypertension in older adults in Africa?

3. What is the level of awareness of high blood pressure among older adults in Africa?

\section{Methods}

\section{Protocol and registration}

This protocol complies with the Preferred Reporting Items for Systematic Reviews and Meta-analyses (PRISMA-P) guidelines (Additional file 1) [52]. It is registered on PROSPSERO international prospective register of systematic reviews (http://www.crd.york.ac.uk/PROSPERO), registration number CRD42017056474 [53]. In the event of any amendment to this protocol post-publication, then the date and the rationale for the amendments will be published in the PROSPERO register in a way that enables these changes to be tracked.

\section{Inclusion criteria}

The following criteria should be met by studies to be included in the analysis:

1. Types of studies: population-based cross-sectional studies and follow-up studies published from January 1980 to present which report prevalence or incidence of hypertension in older adults.

2. Types of articles: both published and unpublished articles will be eligible for inclusion. Conference abstracts which provide adequate information on the sample size, data collection methods and analysis as well as the prevalence of hypertension will be considered.

3. Population: apparently healthy adults aged 50 years and older living in Africa

4. Outcome measures: the main outcome measure is the prevalence or incidence of hypertension.
Secondary outcomes include the grades of hypertension, the proportion of hypertensives detected and the presence of co-morbidities.

5. Setting/context: only studies of persons living in Africa will be included. Multi-country or multi-region studies will be included if there is distinct analysis of data pertaining to African countries.

\section{Exclusion criteria}

We will exclude the following studies:

1. Type of population:

a. Studies involving older adults in a restricted population such as those who are unwell, attending hospitals or are in institutions for chronic or mental illness

b. Studies involving older persons in refugee settings

c. African migrants living outside the African region

2. Types of articles: Review articles, case reports and expert opinion commentaries

3. Type of outcome measure: studies whose main outcome is self-reported hypertension or nonsystemic hypertension such as pulmonary hypertension

4. Geographical area: studies conducted outside the African region

\section{Literature search}

We will search the major electronic databases (EMBASE and MEDLINE via Ovid and Academic Search Complete, CINAHL and PsycINFO via EBSCOhost) and repositories (African Index Medicus and African Journals Online). Using the PICO's approach $[54,55]$, we will search the literature based on four concepts: the population (older adults), the type of measure (prevalence or incidence), outcome (hypertension or raised blood pressure) and the geographic setting (Africa). In EMBASE and MEDLINE databases, the search terms relating to three concepts will include major medical subject headings (MeSH) and freetext words (Table 1). The Boolean operators 'OR' will be used with synonyms within each concept and then the search results for the different concepts combined with the 'AND' operator. The aim is to maximise the yield of the relevant papers. Some of the search terms will be used in truncated formats to permit wider and different sets of the term to be applied simultaneously. Regarding the geographic context (Africa), the different regions and the individual countries of the continent will be entered separated by the 'OR' operator.

Regarding the fourth concept, the study population, we will apply the limits available in the database to select the subset of studies pertaining to those aged 50 years and older. Besides the proceedings and abstracts from conference that will be obtained from the above 
Table 1 Search method in EMBASE and Ovid MEDLINE

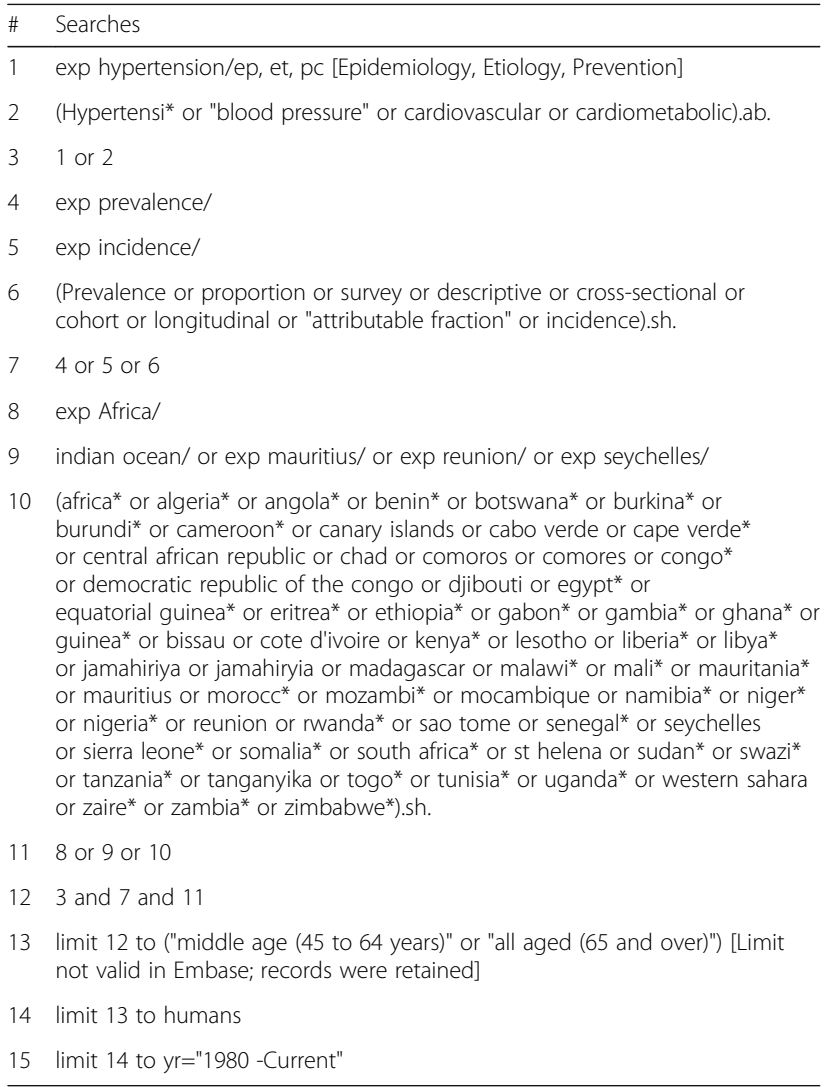

databases, we will search Google Scholar and ProQuest for other grey literature presented as dissertations or unpublished reports. Where possible, we will contact the authors for the full results of conference abstracts. The bibliographies of the selected papers that the selected databases yield will also be hand-searched to locate further articles of interest.

\section{Selection of studies}

Duplicate studies from the different electronic databases, which emerge after using the above search strategy, will be removed through the EndNote programme. The titles and abstracts of the articles of results that emerge from the search strategy will be screened independently by two reviewers to exclude obviously non-relevant papers (Fig. 1). Then the full-text versions of the remaining potentially eligible articles will be retrieved and further assessed independently by two reviewers to determine if they satisfy the inclusion criteria. Any discrepancies will be resolved through consensus. Corresponding authors will be contacted to provide copies of papers whose full text is not available online. In line with the PRISMA framework, the reasons for exclusion at full-text screen level will be documented (Fig. 1) [52].

The total number of unique studies from all sources which fulfill the inclusion criteria will be recorded. The list of studies that were excluded from analysis and the reasons for their exclusion will be reported as supplementary information.

\section{Data extraction}

Data will be extracted using a standardised extraction form in Microsoft Excel (Additional file 2). The data to be captured will include the publication details, language of the paper, study period, study location, study objectives, geographic setting, study design, study period, characteristics of participants, sample size and sampling technique, explanatory and outcome variables, data analysis and the major findings. Specific data such as the demographic and socio-economic characteristics, any anthropometric measurements, blood pressure measurement procedures, the overall prevalence and grades of hypertension, and the effect sizes of statistically significant variables will be extracted. The variables that are associated with hypertension will be categorised into psychosocial, economic, social, dietary, behavioural and lifestyle factors [56]. Where multiple papers pertaining to the same study population and site are encountered, we will extract data from the most informative paper(s) but they will be linked together as one unique study paper. The earliest year of publication will be adopted if multiple papers of the same study are published at different times.

In line with the Seventh Report of the Joint National Committee on the Prevention, Detection, Evaluation and Treatment of High Blood Pressure (JNC VII), hypertension will be defined as having a SBP $\geq 140 \mathrm{mmHg}$ and/ or diastolic pressure (DBP) $\geq 90 \mathrm{mmHg}$ or using antihypertensive drug therapy in the previous 2 weeks [57]. Those who report being told of a previous diagnosis of hypertension by a health professional would be considered as being aware of their hypertension. The three grades of hypertension will be defined as grade 1 (SBP 140-159 mmHg or DBP 90-99 $\mathrm{mmHg}$ ), grade 2 (SBP 160-179 $\mathrm{mmHg}$ or DBP $100-109 \mathrm{mmHg}$ ) and grade 3 (SBP $\geq 180 \mathrm{mmHg}$ or $\mathrm{DBP} \geq 110 \mathrm{mmHg}$ ) [58]. The number of risk factors in the same individuals and the presence of co-morbidities such as diabetes and chronic kidney disease will be documented.

\section{Assessment of the quality of studies}

The quality of included studies will be assessed using a risk of bias tool specifically designed and validated for cross-sectional studies [59]. The tool assesses both internal and external validity based on responses to ten questions. As with the study selection, two different investigators will independently evaluate the quality of the included papers and characterize them as having a high, moderate or low risk of bias. Any discrepancies will be resolved by mutual consent and discussion. 


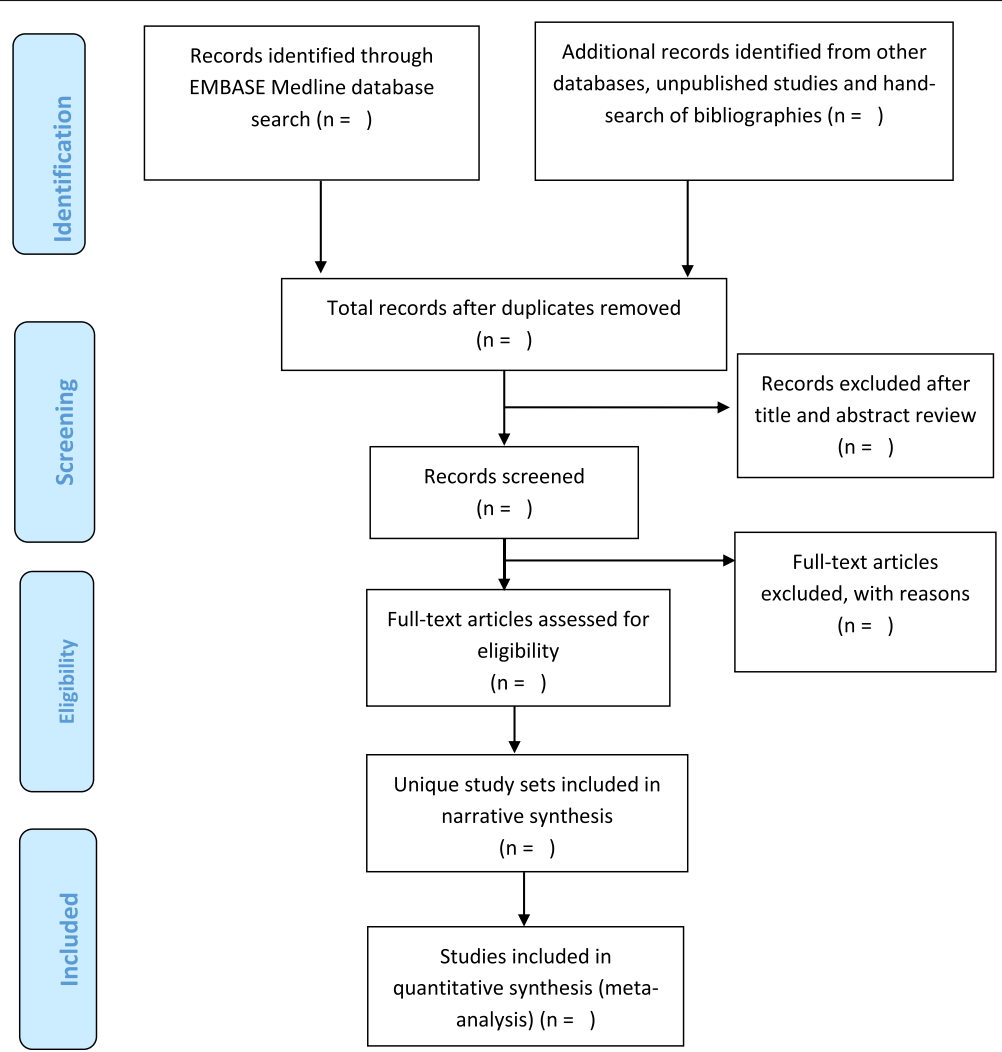

Fig. 1 Flow chart for selection of papers. Charts the selection of articles in the review in line with the Preferred Reporting Items for Systematic Review and Meta-analysis (PRISMA) Framework

\section{Data analysis and synthesis}

The characteristics of the study participants will be summarised in tables using descriptive statistics. The main outcomes are hypertension, the grades of hypertension, and the proportion of controlled hypertension. The strength of the association between these outcomes and the various demographic, socio-economic and lifestyle factors in bivariate and multivariable analysis will be reported. The confounders adjusted in each analysis will be documented for each study.

The prevalence data and risk factors from the different studies will be summarised using narrative synthesis. The prevalence will be summarised as a proportion, and summary measures of risk factor-hypertension association, presented as odds ratios. If statistically appropriate, prevalence estimates from studies with common definitions of hypertension will be pooled together to provide a single summary estimate through a randomeffects meta-analysis [60].

We will report the crude and pooled estimates of the prevalence of hypertension in 10-year time periods over the period 1980-2017 in order to provide trends [19, 41]. In addition, we will analyse the temporal trends in the prevalence of hypertension using cumulative metaanalysis $[46,61]$.
We will explore heterogeneity through the inspection of forest plots as well as with the chi-squared test on Cochran's Q (alpha set at 0.1) statistic [62] and an $I^{2}$ test. For the latter, the cut-points for low, moderate and high degrees of heterogeneity will be set at 25, 50 and $75 \%$ respectively [63]. Separate sub-group analyses will be performed to assess the causes of any heterogeneity and, if statistically appropriate, to estimate the pooled prevalence of hypertension by sex, age group, geographic locality, study design and year of publication (by decades). We will perform a sensitivity analysis to examine the effect of excluding studies with high-risk of bias on the overall prevalence and relationships. The presence of reporting bias will be determined through funnel plot asymmetry if more than 10 eligible studies are included and Egger's test [64]. Data analysis will be performed using the Stata version 14 and RevMan 5.3 programmes.

The overall quality of evidence will be based on an adaptation of the Grading of Recommendations Assessment, Development and Evaluation (GRADE) guidelines [65]. The GRADE criteria for the evaluation of evidence are risk of bias, consistency (heterogeneity), directness (generalizability), precision (statistical significance of effect measures) and publication bias. The quality of evidence is graded as high, moderate, low or very low. For 
observational studies, the quality of evidence starts off as low [66]. The risk of bias will be derived from the Hoy et al. tool designed for prevalence studies [59].

\section{Discussion}

To our knowledge, this is the first published protocol to systematically estimate the prevalence of hypertension and its associated factors in the adult population aged 50 years and older in Africa. Furthermore, our review has several other strengths. The search strategy is comprehensive in including published and unpublished literature. The four-decade period covered by the review is sufficiently long to establish patterns and relationships with the outcome. The reviewers recognize that bias can occur even in apparently well-conducted studies [67]. Hence, the review will evaluate the risk of bias in the primary studies. In order to reduce bias, two researchers will independently screen and agree on the study papers to be included and assess their quality [52, 68]. To ensure maximum yield, no language restrictions will be applied.

As with other reviews [21, 44, 69], we do not specifically include 'awareness' and 'risk factors' in the search terms. The heterogeneity in the study population, blood pressure measurement protocols and study methods may limit the meta-analysis or generalizability. Narrative synthesis would be used if meta-analysis is not appropriate. Another limitation is that most of the studies will be cross-sectional and so will not be best suited to assess temporal sequence between a risk factor and the outcome.

The findings will be submitted for publication in peer-reviewed open access journals as well for presentation at a scientific conference. They will be shared with the health departments of the African Union and the Regional Economic Communities of Africa to promote discussion among regional and national policy makers. The paper will add to the knowledge of the burden of hypertension and its associated lifestyle and behavioural practices in this group of older adults who are largely neglected in many African countries. The findings will assist countries to commit to favourable policies and programmes for healthy ageing, in line with the World Health Organization's global strategy on ageing and health [70].

This protocol presents a comprehensive and reproducible approach to systematically estimate the prevalence of hypertension in the older adult population of Africa. It tries to conform with the best practices at each stage of the protocol development in order to assure highquality output. It is expected that the findings of the systematic review would galvanize national and regional efforts to improve the cardiovascular health of this often-neglected population in Africa.

\section{Additional files}

Additional file 1: PRISMA-P 2015 checklist 0108 2017. Description of data: Contains the line numbers for the different items on the PRISMA-P checklist. (DOCX 33 kb)

Additional file 2: Data extraction form HTN protocol. Data extraction form for studies on the prevalence of hypertension in older adults in Africa. Contains the data extraction form. (DOCX 38 kb)

\section{Abbreviations}

BP: Blood pressure; DALY: Disability-adjusted life years; DBP: Diastolic blood pressure; JNC: Joint National Committee; NCDs: Non-communicable diseases; PRISMA: Preferred Reporting Items for Systematic Review and Meta-Analysis; PROSPERO: International prospective register of systematic reviews; SBP: Systolic blood pressure; SSA: Sub-Saharan Africa; WHO: World Health Organization

\section{Acknowledgements}

We are grateful to the reviewers for their useful comments. We thank our respective organizations for the opportunity to undertake this study.

\section{Funding}

The authors received no specific funding for this study.

\section{Availability of data and materials}

Data sharing is not applicable to this article as no datasets were generated or analysed during the current study. The data extraction form is presented as supplementary information.

\section{Authors' contributions}

All authors contributed to the design of the protocol. WKB conceived the study and wrote the protocol under the supervision of SR and EZ. All authors read and approved the final manuscript. WKB is the guarantor of this protocol.

\section{Ethics approval and consent to participate}

Not applicable.

\section{Consent for publication}

Not applicable.

\section{Competing interests}

The authors declare that they have no competing interests. Our respective employers had no influence in the development of this review protocol.

\section{Publisher's Note}

Springer Nature remains neutral with regard to jurisdictional claims in published maps and institutional affiliations.

\section{Author details}

${ }^{1}$ West African Health Organisation (WAHO), 175 Avenue Ouezzin Coulibaly, 01 BP 153, Bobo-Dioulasso, Burkina Faso. 'School of Public Health, University of Ghana, LG 13, Legon, Accra, Ghana. ${ }^{3}$ Division of Health Research, Faculty of Health \& Medicine, Lancaster University, Furness Building, Lancaster LA1 $4 Y G$, UK.

Received: 9 May 2017 Accepted: 22 September 2017

Published online: 04 October 2017

References

1. O'Donnell MJ, Chin SL, Rangarajan S, Xavier D, Liu L, Zhang H, Rao-Melacini $P$, Zhang $X$, Pais P, Agapay S. Global and regional effects of potentially modifiable risk factors associated with acute stroke in 32 countries (INTERSTROKE): a case-control study. Lancet. 2016:388(10046):761-75.

2. World Health Organization. World Health Statistics 2014. Geneva: WHO; 2014

3. NCD Risk Factor Collaboration. Worldwide trends in blood pressure from 1975 to 2015: a pooled analysis of 1479 population-based measurement studies with 19.1 million participants. Lancet. 2016; doi: 10.1016/s01406736(16)31919-5. 
4. Ekwunife O, Aguwa C. PCV 27: Increasing prevalence of hypertension in Nigeria: a systematic review from 1990 to 2009. Value Health. 2010;13(7): A345

5. Fezeu L, Kengne AP, Balkau B, Awah PK, Mbanya JC. Ten-year change in blood pressure levels and prevalence of hypertension in urban and rural Cameroon. J Epidemiol Community Health. 2010;64(4):360-5.

6. Okeahialam BN, Ogbonna C, Otokwula AE, Joseph DE, Chuhwak EK, Isiguzoro 1O. Cardiovascular epidemiological transition in a rural habitat of Nigeria: the case of Mangu Local Government Area. West Afr J Med. 2012; 31(1):14-8.

7. Mensah GA. Epidemiology of stroke and high blood pressure in Africa. Heart. 2008;94(6):697-705.

8. Addo J, Smeeth L, Leon DA. Hypertensive target organ damage in Ghanaian civil servants with hypertension. PLoS One. 2009;4(8):e6672.

9. Okpechi I, Oluleye TS, Bekibele CO, Adedapo KS, Adebiyi AA, Ogah OS, Salako BL. Unexpectedly high prevalence of target-organ damage in newly diagnosed Nigerians with hypertension : cardiovascular topic. Cardiovasc J South Afr. 2007;18(2):77-83.

10. Oladapo OO, Salako L, Sadiq L, Shoyinka K, Adedapo K, Falase AO. Targetorgan damage and cardiovascular complications in hypertensive Nigerian Yoruba adults: a cross-sectional study. Cardiovasc J Afr. 2012;23(7):379-84.

11. Nelissen HE, Hendriks ME, Wit FW, Bolarinwa OA, Osagbemi GK, Bindraban NR, Lange JM, Akande TM, Schultsz C, Brewster LM. Target organ damage among hypertensive adults in rural Nigeria: a cross-sectional study. J Hypertens. 2014;32(3):487-94.

12. Katchunga P, Malanda B, Mweze M, Dupont B, M'Buyamba-Kabangu J, Kashongwe Z, Kabinda J, Buysschaert M: Knowledge of the general population about hypertension and diabetes mellitus in South Kivu, Democratic Republic of Congo. Rev Epidemiol Santé Publique 2012, 60(2): 141-147.

13. Mugwano I, Kaddumukasa M, Mugenyi L, Kayima J, Ddumba E, Sajatovic M, Sila C, DeGeorgia M, Katabira E. Poor drug adherence and lack of awareness of hypertension among hypertensive stroke patients in Kampala, Uganda: a cross sectional study. BMC Res Notes. 2016;9:3.

14. Lopes Ibanez-Gonzalez D. Perspectives on healthcare, chronic noncommunicable disease, and healthworlds in an urban and rural setting. Glob Health Action. 2014;7:25317.

15. Naanyu V, Vedanthan R, Kamano JH, Rotich JK, Lagat KK, Kiptoo P, Kofler C, Mutai KK, Bloomfield GS, Menya D, et al. Barriers Influencing Linkage to Hypertension Care in Kenya: Qualitative Analysis from the LARK Hypertension Study. J Gen Intern Med. 2016;31(3):304-14.

16. Seedat $Y$. Why is control of hypertension in sub-Saharan Africa poor? Cardiovasc J Afr. 2015;26(4):193.

17. Kayima J, Wanyenze RK, Katamba A, Leontsini E, Nuwaha F. Hypertension awareness, treatment and control in Africa: a systematic review. BMC Cardiovasc Disord. 2013;13:54

18. Mugomeri E, Ramathebane MV, Maja L, Chatanga P, Moletsane L. Knowledge of disease condition and medications among hypertension patients in Lesotho. J Am Soc Hypertens. 2016;10(1):41-6.

19. Bosu WK. The prevalence, awareness, and control of hypertension among workers in West Africa: a systematic review. Glob Health Action. 2015;8: 26227.

20. Sarki AM, Nduka CU, Stranges S, Kandala N-B, Uthman OA. Prevalence of Hypertension in Low-and Middle-Income Countries: A Systematic Review and Meta-Analysis. Medicine. 2015:94(50):e1959.

21. Adeloye D, Basquill C. Estimating the prevalence and awareness rates of hypertension in Africa: a systematic analysis. PLoS One. 2014;9(8):e104300.

22. Duda RB, Anarfi JK, Adanu RM, Seffah J, Darko R, Hill AG. The health of the "older women" in Accra, Ghana: results of the Women's Health Study of Accra. J Cross Cult Gerontol. 2011;26(3):299-314.

23. Negin J, Cumming R, de Ramirez SS, Abimbola S, Sachs SE. Risk factors for non-communicable diseases among older adults in rural Africa. Tropical Med Int Health. 2011;16(5):640-6.

24. Abegunde KA, Owoaje ET. Health problems and associated risk factors in selected urban and rural elderly population groups of South-West Nigeria. Ann Afr Med. 2013;12(2):90.

25. Arokiasamy $P$, Uttamacharya U, Jain K, Biritwum RB, Yawson AE, Wu F, Guo $Y$, Maximova T, Espinoza BM, Rodríguez AS. The impact of multimorbidity on adult physical and mental health in low-and middle-income countries: what does the study on global ageing and adult health (SAGE) reveal? BMC Med. 2015;13(1):178.
26. Garin N, Koyanagi A, Chatterji S, Tyrovolas S, Olaya B, Leonardi M, Lara E, Koskinen S, Tobiasz-Adamczyk B, Ayuso-Mateos JL. Global multimorbidity patterns: a cross-sectional, population-based, multi-country study. J Geronto A Biol Sci Med Sci. 2016;71(2):205-14

27. Hien H, Berthe A, Drabo MK, Meda N, Konate B, Tou F, Badini-Kinda F, Maca J. Prevalence and patterns of multimorbidity among the elderly in Burkina Faso: cross-sectional study. Tropical Med Int Health. 2014;19(11):1328-33.

28. Akinyemi RO, Izzeldin IM, Dotchin C, Gray WK, Adeniji O, Seidi OA, Mwakisambwe JJ, Mhina CJ, Mutesi F, Msechu HZ, et al. Contribution of noncommunicable diseases to medical admissions of elderly adults in Africa: a prospective, cross-sectional study in Nigeria, Sudan, and Tanzania. J Am Geriatr Soc. 2014;62(8):1460-6.

29. Hammami S, Mehri S, Hajem S, Koubaa N, Frih MA, Kammoun S, Hammami $\mathrm{M}$, Betbout F. Awareness, treatment and control of hypertension among the elderly living in their home in Tunisia. BMC Cardiovasc Disord. 2011;11(1):65.

30. Yang F, Qian D, Hu D. Prevalence, awareness, treatment, and control of hypertension in the older population: results from the multiple national studies on ageing. J Am Soc Hypertens. 2016;10(2):140-8.

31. Macia E, Duboz P, Gueye L. Prevalence, awareness, treatment and control of hypertension among adults 50 years and older in Dakar, Senegal. Cardiovasc J Afr. 2012:23(5):265-9.

32. United Nations. World Population Prospects: The 2017 Revision, custom data acquired via website. New York: Department of Economic and Social Affairs, Population Division; 2017

33. United Nations. Sub-Saharan Africa's growing population of older persons. Population Facts. 2016;1:1-2

34. IHME. Global Burden of Disease (GBD) Compare Data Visualization, vol. 2016 Seattle: Institute for Health Metrics and Evaluation, University of Washington; 2016.

35. Rowe JW, Kahn RL. Successful aging. Gerontologist. 1997;37(4):433-40.

36. Wannamethee SG, Shaper AG, Walker M. Changes in physical activity, mortality, and incidence of coronary heart disease in older men. Lancet. 1998;351(9116):1603-8

37. James PA, Oparil S, Carter BL, Cushman WC, Dennison-Himmelfarb C, Handler J, Lackland DT, LeFevre ML, MacKenzie TD, Ogedegbe O, et al. 2014 evidence-based guideline for the management of high blood pressure in adults: report from the panel members appointed to the Eighth Joint National Committee (JNC 8). JAMA. 2014:311(5):507-20.

38. Ettehad D, Emdin CA, Kiran A, Anderson SG, Callender T, Emberson J, Chalmers J, Rodgers A, Rahimi K. Blood pressure lowering for prevention of cardiovascular disease and death: a systematic review and meta-analysis. Lancet. 2016;387(10022):957-67.

39. Adeloye D, Basquill C, Aderemi AV, Thompson JY, Obi FA. An estimate of the prevalence of hypertension in Nigeria: a systematic review and metaanalysis. J Hypertens. 2015;33(2):230-42.

40. Bosu WK. Epidemic of hypertension in Ghana: a systematic review. BMC Public Health. 2010;10:418.

41. Ogah OS, Okpechi I, Chukwuonye II, Akinyemi JO, Onwubere BJ, Falase AO, Stewart S, Sliwa K. Blood pressure, prevalence of hypertension and hypertension related complications in Nigerian Africans: A review. World $J$ Cardiol. 2012;4(12):327-40.

42. Akinlua JT, Meakin R, Umar AM, Freemantle N. Current Prevalence Pattern of Hypertension in Nigeria: A Systematic Review. PLoS One. 2015;10(10): e0140021.

43. Addo J, Smeeth L, Leon DA. Hypertension in sub-saharan Africa: a systematic review. Hypertension. 2007;50:1012-8.

44. Ataklte F, Erqou S, Kaptoge S, Taye B, Echouffo-Tcheugui JB, Kengne AP. Burden of undiagnosed hypertension in Sub-Saharan Africa A systematic review and meta-analysis. Hypertension. 2015;65(2):291-8.

45. Essouma M, Noubiap JJN, Bigna JJR, Nansseu JRN, Jingi AM, Aminde LN, Zafack J. Hypertension prevalence, incidence and risk factors among children and adolescents in Africa: a systematic review and meta-analysis protocol. BMJ Open. 2015;5(9):e008472.

46. Kaze AD, Schutte AE, Erqou S, Kengne AP, Echouffo-Tcheugui JB. Prevalence of hypertension in older people in Africa: a systematic review and metaanalysis. J Hypertens. 2017; doi: 10.1097/hih.0000000000001345.

47. Wu F, Guo Y, Chatterji S, Zheng Y, Naidoo N, Jiang Y, Biritwum R, Yawson A, Minicuci N, Salinas-Rodriguez A, et al. Common risk factors for chronic noncommunicable diseases among older adults in China, Ghana, Mexico, India, Russia and South Africa: the study on global AGEing and adult health (SAGE) wave 1. BMC Public Health. 2015;15:88. 
48. Addo J, Amoah AGB, Koram KA. The changing patterns of hypertension in Ghana: a study of four rural communities in the Ga district. Ethn Dis. 2006; 16:894-9.

49. Addo J, Smeeth L, Leon DA. Prevalence, detection, management, and control of hypertension in Ghanaian civil servants. Ethn Dis. 2008;18:505-11.

50. Peltzer K, Phaswana-Mafuya N. Hypertension and associated factors in older adults in South Africa. Cardiovasc J Afr. 2013;24(3):67-72.

51. Doulougou B, Kouanda S, Rossier C, Soura A, Zunzunegui MV. Differences in hypertension between informal and formal areas of Ouagadougou, a subSaharan African city. BMC Public Health. 2014;14:893.

52. Shamseer L, Moher D, Clarke M, Ghersi D, Liberati A, Petticrew M, Shekelle P, Stewart LA. Preferred reporting items for systematic review and metaanalysis protocols (PRISMA-P) 2015: elaboration and explanation. BMJ. 2015; 349:97647.

53. Booth A, Clarke M, Dooley G, Ghersi D, Moher D, Petticrew M, Stewart L. The nuts and bolts of PROSPERO: an international prospective register of systematic reviews. Syst Rev. 2012;1:2.

54. O'Connor AM, Anderson KM, Goodell CK, Sargeant JM. Conducting systematic reviews of intervention questions I: Writing the review protocol, formulating the question and searching the literature. Zoonoses Public Health. 2014;61(Suppl 1):28-38.

55. Moher D, Shamseer L, Clarke M, Ghersi D, Liberati A, Petticrew M, Shekelle P, Stewart LA. Preferred reporting items for systematic review and metaanalysis protocols (PRISMA-P) 2015 statement. Syst Rev. 2015:4(1):1.

56. Mayega RW, Makumbi F, Rutebemberwa E, Peterson S, Östenson C-G, Tomson G, Guwatudde D. Modifiable Socio-Behavioural Factors Associated with Overweight and Hypertension among Persons Aged 35 to 60 Years in Eastern Uganda. PLoS One. 2012;7(10):e47632.

57. Chobanian A, Bakris GL, Black H, Cushman WC, Green LA, Izzo J, Jones D, Materson B, Oparil S, Wright JT, et al. The Seventh Report of the Joint National Committee on Prevention, Detection, Evaluation, and Treatment of High Blood Pressure: the JNC 7 report. JAMA. 2003;289:2560-72.

58. Whitworth JA. 2003 World Health Organization (WHO)/International Society of Hypertension (ISH) statement on management of hypertension. J Hypertens. 2003;21(11):1983-92.

59. Hoy D, Brooks P, Woolf A, Blyth F, March L, Bain C, Baker P, Smith E, Buchbinder R. Assessing risk of bias in prevalence studies: modification of an existing tool and evidence of interrater agreement. J Clin Epidemiol. 2012;65(9):934-9.

60. DerSimonian R, Laird N. Meta-analysis in clinical trials. Control Clin Trials. 1986;7(3):177-88.

61. Lau J, Schmid CH, Chalmers TC. Cumulative meta-analysis of clinical trials builds evidence for exemplary medical care. J Clin Epidemiol. 1995;48(1):45-57.

62. Cochran WG. The combination of estimates from different experiments. Biometrics. 1954;10(1):101-29.

63. Higgins JP, Thompson SG, Deeks JJ, Altman DG. Measuring inconsistency in meta-analyses. BMJ. 2003;327(7414):557-60.

64. Egger M, Davey Smith G, Schneider M, Minder C. Bias in meta-analysis detected by a simple, graphical test. BMJ. 1997;315(7109):629-34.

65. Balshem $H$, Helfand $M$, Schunemann HJ, Oxman AD, Kunz R, Brozek J, Vist GE, Falck-Ytter Y, Meerpohl J, Norris S, et al. GRADE guidelines: 3. Rating the quality of evidence. J Clin Epidemiol. 2011;64(4):401-6.

66. Guyatt G, Oxman AD, Akl EA, Kunz R, Vist G, Brozek J, Norris S, Falck-Ytter Y, Glasziou P, Jaeschke R. GRADE guidelines: 1. Introduction-GRADE evidence profiles and summary of findings tables. J Clin Epidemiol. 2011;64(4):383-94.

67. Higgins JPT, Green S. Cochrane Handbook for Systematic Reviews of Interventions Version 5.1.0, vol. 2017: The Cochrane Collaboration; 2011.

68. Buscemi N, Hartling L, Vandermeer B, Tjosvold L, Klassen TP. Single data extraction generated more errors than double data extraction in systematic reviews. J Clin Epidemiol. 2006;59(7):697-703.

69. Tailakh A, Evangelista LS, Mentes JC, Pike NA, Phillips LR, Morisky DE. Hypertension prevalence, awareness, and control in Arab countries: a systematic review. Nurs Health Sci. 2014;16(1):126-30

70. WHO. Global strategy and action plan on ageing and health (2016-2020). Geneva: World Health Organization; 2016.

\section{Submit your next manuscript to BioMed Central and we will help you at every step:}

- We accept pre-submission inquiries

- Our selector tool helps you to find the most relevant journal

- We provide round the clock customer support

- Convenient online submission

- Thorough peer review

- Inclusion in PubMed and all major indexing services

- Maximum visibility for your research

Submit your manuscript at www.biomedcentral.com/submit
Biomed Central 